\title{
AN EXAMPLE IN THE SPACE OF BOUNDED OPERATORS FROM $C(X)$ TO $C(Y)$
}

\author{
SAMUEL KAPLAN
}

\begin{abstract}
Let $X, Y$ be compact spaces, and $C(X), C(Y)$ the corresponding Banach lattices of real continuous functions. It is shown that the partially ordered space of bounded operators from $C(X)$ to $C(Y)$ need not be generated by its positive cone.
\end{abstract}

1. In the following $X, Y$ are compact spaces, and $C(X), C(Y)$ the Banach lattices of real continuous functions on $X, Y$ respectively. A linear mapping $T: C(X) \rightarrow C(Y)$ is norm-bounded if and only if it is order-bounded, hence we can simply use the word bounded. We denote by $B(C(X), C(Y))$ the space of all bounded linear mappings of $C(X)$ into $C(Y)$, and we endow it with the canonical order, determined by taking for positive cone the set of all positive linear mappings.

U. Krengel has given an example [1] to show that $B(C(X), C(Y))$ may fail to be a Riesz space. In the present note we modify his example to show that $B(C(X), C(Y))$ may even fail to be generated by its positive cone.

We first give a simplification of Krengel's example. Let $X$ be $\alpha N$, the Alexandroff one-point compactification of the set $N$ of natural numbers: $X=\{1,2, \cdots, n, \cdots, \omega\}$. Define $T: C(X) \rightarrow C(X)$ as follows: for each $f \in C(X), T f$ is the element of $C(X)$ with values

$$
\begin{aligned}
(T f)(2 n-1) & =f(2 n-1)-f(2 n) & & (n=1,2, \cdots), \\
(T f)(2 n) & =0 & & (n=1,2, \cdots), \\
(T f)(\omega) & =0 . & &
\end{aligned}
$$

It is immediate that $T \in B(C(X), C(X))$. We show $T^{+}$does not exist in $B(C(X), C(X))$, that is, there is no smallest positive linear mapping above $T$. Suppose a mapping $U$ satisfies $U \geqq 0, U \geqq T$. We have to produce a mapping $V$ such that $0 \leqq V<U, V \geqq T$.

It is easily verified that

$$
f \geqq 0 \quad \text { implies } \quad(U f)(2 n-1) \geqq f(2 n-1) \text { for all } n .
$$

Received by the editors September 19, 1972.

AMS (MOS) subject classifications (1970). Primary 46A40, 46E05, 47B55.

Key words and phrases. Continuous functions, bounded linear mappings, positive cone.

(c) American Mathematical Society 1973 
In particular, denoting by $\mathbf{1}$ the constant function of value 1 , (U1) $(2 n-1) \geqq 1$ for all $n$. Since $U 1$ is continuous, it follows that for $n$ large enough, $(U 1)(2 n)>0$. Choose such a large $n_{0}$ and let $P$ be the projection in $C(X)$ which for every $g \in C(X)$, sends $g\left(2 n_{0}\right)$ into 0 . The mapping $P \circ U$ is then the desired $V$.

2. We turn to our example of a $T \in B(C(X), C(Y))$ which has no positive mapping above it. Let $\left\{X_{n}\right\}(n=1,2, \cdots)$ be a sequence of copies of $\alpha N$,

$$
X_{n}=\left\{x_{n 1}, x_{n 2}, \cdots, x_{n m}, \cdots, x_{n \omega}\right\} \quad(n=1,2, \cdots),
$$

$\sum_{n} X_{n}$ their topological sum, and set $X=\alpha\left(\sum_{n} X_{n}\right)$, with the adjoined element denoted by $x_{\omega}$.

Let $\left\{Y_{n}\right\}(n=1,2, \cdots)$ be a sequence of copies of $N$,

$$
Y_{n}=\left\{y_{n 1}, y_{n 2}, \cdots, y_{n m}, \cdots\right\} \quad(n=1,2, \cdots),
$$

$\sum_{n} Y_{n}$ their topological sum, and set $Y=\alpha\left(\sum_{n} Y_{n}\right)$, with the adjoined element denoted by $y_{\omega}$. (Thus $Y$ is simply $\alpha N$ with $N$ decomposed into an infinite number of disjoint infinite subsets.)

Define $T: C(X) \rightarrow C(Y)$ as follows: for each $f \in C(X), T f$ is the element of $C(Y)$ with values

$$
\begin{aligned}
(T f)\left(y_{n m}\right) & =f\left(x_{n, 2 m-1}\right)-f\left(x_{n, 2 m}\right) \quad(n, m=1,2, \cdots) \\
(T f)\left(y_{\omega}\right) & =0 .
\end{aligned}
$$

We first verify that $T f \in C(Y)$, that is, it is continuous at $y_{\omega}$. Given $\varepsilon>0$, there exists $n_{0}$ such that for $n>n_{0}$,

$$
\left|f\left(x_{n, m}\right)-f\left(x_{\omega}\right)\right|<\varepsilon / 2 \text { for all } m,
$$

whence $\left|f\left(x_{n .2 m-1}\right)-f\left(x_{n, 2 m}\right)\right|<\varepsilon$ for all $m$. Having chosen $n_{0}$, choose $m_{0}$ such that for $n=1, \cdots, n_{0}$ and $m>m_{0},\left|f\left(x_{n, m}\right)-f\left(x_{n \omega}\right)\right|<\varepsilon / 2$, whence $\left|f\left(x_{n, 2 m-1}\right)-f\left(x_{n, 2 m}\right)\right|<\varepsilon$. Thus for all $y_{n m}$ outside the finite set $\left\{y_{n m} \mid n=1, \cdots, n_{0} ; m=1, \cdots, m_{0}\right\},\left|(T f)\left(y_{n m}\right)\right|<\varepsilon$.

$T$ is clearly linear, and is bounded (in effect, $\|T\|=2$ ).

Suppose finally, that there exists $U \geqq 0, U \geqq T$. Again denoting by 1 the constant function on $X$ of value 1 , we show that $(U 1)\left(y_{\omega}\right) \geqq k$ for all positive integers $k$, giving a contradiction.

For each $n$, let $e_{n}$ be the characteristic function of the subset $X_{n}$ of $X$.

$$
\text { For every } n=1,2, \cdots, \quad\left(U e_{n}\right)\left(y_{\omega}\right) \geqq 1 .
$$

Fix $n$. As in (2) above, it is easy to verify that for every $m,\left(U e_{n}\right)\left(y_{n m}\right) \geqq$ $e_{n}\left(x_{n, 2 m-1}\right)=1$. Since $U e_{n}$ is continuous, this gives (i). 
1973] THE SPACE OF BOUNDED OPERATORS FROM $C(X)$ TO $C(Y)$

Now given $k, 1 \geqq \sum_{1}^{k} e_{n}$, whence $U 1 \geqq U\left(\sum_{1}^{k} e_{n}\right)=\sum_{1}^{k} U e_{n}$, whence finally, $(U 1)\left(y_{\omega}\right) \geqq \sum_{1}^{k}\left(U e_{n}\right)\left(y_{\omega}\right) \geqq k$.

\section{REFERENCE}

1. Ulrich Krengel, Über den Absolutbetrag stetiger linearer Operatoren und seine Anwendung auf ergodische Zerlegungen, Math. Scand. 13 (1963), 151-187. MR 31 \#310.

Department of Mathematics, Purdue University, West lafayette, Indiana 47907 